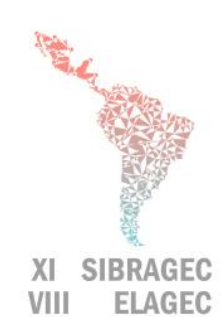

VIII ELAGEC

\section{SIMPÓSIO BRASILEIRO DE GESTÃO E ECONOMIA DA CONSTRUÇÃO \\ VIII ENCUENTRO LATINOAMERICANO DE GESTIÓN \\ Y ECONOMÍA DE LA CONSTRUCCIÓN}

Do conhecimento à ação: práticas avançadas de gestão da produção

Londrina, Paraná, Brasil. 23 a 25 de Outubro de 2019

\title{
NANOMATERIALES EN OBRA DE CONSTRUCCIÓN: ESTADO ACTUAL DE LA PREVENCIÓN DE RIESGOS LABORALES
}

\author{
DÍAZ-SOLER, Beatriz (1); LÓPEZ-ALONSO, Mónica (2); MARTÍNEZ-AIRES, \\ $M^{\mathrm{a}}$ Dolores (3)
}

(1) Universidad de Granada (España), email: atriz@ correo.ugr.es (2) Universidad de Granada, email: mlopeza@ugr.es, (3) Universidad de Granada, e-mail: aires@ugr.es

\begin{abstract}
The use of engineered nanomaterials (ENMs), understood as materials whose main constituents present one or more external dimensions in the size range from $1 \mathrm{~nm}$ to $100 \mathrm{~nm}$, allows to improve the properties of a variety of materials and products widely used in the construction sector. Nanotechnology is a key driver for technological innovation in construction sector, where an exponential increase in its application is expected, with some predicting that 50\% of building products will be nano-enabled by 2025. Despite expected growth expectations, its application has been hampered by the current degree of uncertainty regarding the potential effects of NMMs on human health and the environment. This document shows the current status of the recommendations to face this new emerging risk in construction works.
\end{abstract}

Keywords: Nanotechnology, Emerging risk, Construction, Occupational Risk Prevention.

\section{INTRODUCCIÓN}

En la última década, la nanotecnología se ha convertido en una de las áreas de investigación con mayor crecimiento tecnológico y científico, siendo una de las tecnologías con mayor importancia en el desarrollo de nuevos productos.

La nanotecnología puede entenderse como un área científico-técnica dirigida al estudio, diseño, creación, síntesis, manipulación y aplicación de materiales, aparatos y sistemas funcionales a través del control de la materia en la escala del nanómetro (10-9 m), donde las leyes habituales de la física dejan de regir y la física cuántica dispone sus preceptos, manifestándose propiedades ópticas, mecánicas, magnéticas, eléctricas, térmicas y biológicas, diferentes a las habituales (ver Figura 1).

El sector de la construcción no es ajeno a las oportunidades que supone el uso de materiales y productos denominados comúnmente nanomateriales (NMs) o nanomateriales manufacturados (NMMs)con propiedades de alto valor añadido para el sector de la construcción y que, por otro lado, suponen un nuevo reto en la Prevención de los Riegos Laborales. 
SIBRAGEC - ELAGEC 2019 - de 23 a 25 de Outubro - LONDRINA - PR

Figura 1- Introducción a la nanotecnología y NMs

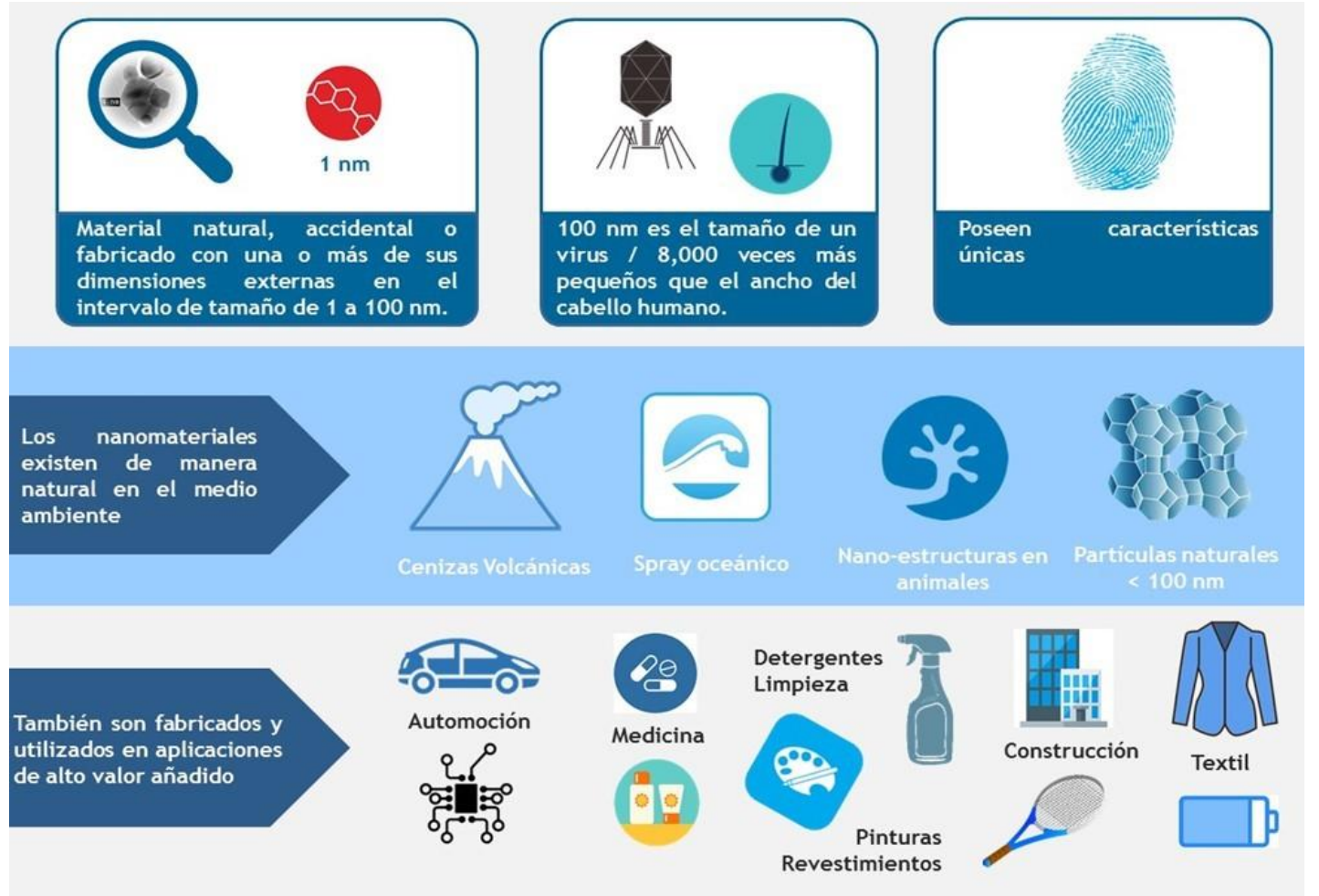

Fuente: (DÍAZ SOLER, 2019)

En este trabajo, se presenta un resumen de NMMs que se utilizan en construcción, así como los niveles de exposición en condiciones previstas de uso e identificar las medidas de control adecuadas para garantizar un alto nivel de protección de la salud de las personas y el medio ambiente.

\section{NMM EN EL SECTOR DE LA CONSTRUCCIÓN}

Aunque comparada con otros sectores aún no han explotado de forma plena todas las nuevas oportunidades que la nanotecnología ofrece, existe un incremento en su aplicación, en este sentido, se prevé un aumento exponencial de su aplicación que se ha estimado podrá suponer que el $50 \%$ de los materiales utilizados en 2025 sean nanoestructurados (AECOM, 2014).

Actualmente, los NMMs más utilizados en este sector son el Dióxido de titanio (TiO2) y el Dióxido de silicio ( $\mathrm{SiO} 2)$. Con menor introducción se encuentran los nanotubos de carbono (CNT), el Grafeno, el Óxido de Hierro (Fe2O3), el Óxido de Aluminio (Al2O3), el Óxido de Zirconio (ZrO2), las Nanofibras y la Plata (Ag) (INSST, 2017; JONES et al., 2015, no 1; STATNANO, 2017).

En la Tabla 1 se pueden observar los NMMs utlizados en los cementos y pinturas, así como las principales propiedades que aportan. Además de estos usos, actualmente se encuentran adhesivos y sellantes, en recubrimientos (de metales, vidrio, madera y hormigón), en ladrillos o pavimentos. 
SIBRAGEC - ELAGEC 2019 - de 23 a 25 de Outubro - LONDRINA - PR

Tabla 1 -NMMs usados em cementos y pinturas. Propriedades que aportan.

\begin{tabular}{|c|c|c|}
\hline Productos & NMMs & Propriedades \\
\hline \multirow{5}{*}{ Cemento } & CNTs & Durabilidad, resistência al agrietamiento, conductividad eléctrica. \\
\hline & $\mathrm{SiO}_{2}$ & $\begin{array}{l}\text { Refuerzo de resistenciamecánica, reducción de lacorrosión, } \\
\text { reducción de lapermeabilidad al agua. }\end{array}$ \\
\hline & $\mathrm{Fe}_{2} \mathrm{O}_{3}$ & $\begin{array}{l}\text { Incremento de lafuerza de compresión, resistencia a laabrasión, } \\
\text { anticorrosión. }\end{array}$ \\
\hline & $\mathrm{TiO}_{2}$ & $\begin{array}{l}\text { Durabilidad, autolimpieza, actividad fotocatalítica en exteriores } \\
\text { (eninvestigaciónpara interiores y bajo luz artificial), rápida } \\
\text { hidratación, incremento del grado de hidratación. }\end{array}$ \\
\hline & Grafeno & $\begin{array}{l}\text { Aumento de impermeabilidad, flexión y plasticidade, } \\
\text { conductividad eléctrica. }\end{array}$ \\
\hline \multirow{5}{*}{ Pinturas } & Plata $(\mathrm{Ag})$ & Actividad biocida. \\
\hline & $\mathrm{TiO}_{2}$ & $\begin{array}{l}\text { Resistencia, actividad fotocatalítica, actividad biocida, } \\
\text { autolimpieza, mantienelatransparencia, hidrofóbico. }\end{array}$ \\
\hline & $\mathrm{SiO}_{2}$ & $\begin{array}{l}\text { Mejoralaadhesión, durabilidade, resistencia al rayado y fácil } \\
\text { limpeza, anti-graffiti. }\end{array}$ \\
\hline & CNTs & Conductividad. \\
\hline & Grafeno & Durabilidad, resistencia, actividad fotocatalítica, actividad biocida \\
\hline
\end{tabular}

Fuente: (INSST, 2017; JONES et al., 2015; STATNANO, 2017).

\section{RIESGOS DE LOS NMS EN EL LUGAR DE TRABAJO}

La exposición a NMMs se produce en todo el ciclo de vida del producto: investigación, proceso productivo, puesta en obra y gestión de residuos.

La vía inhalatoria es la principal vía de entrada de los NMMs en el organismo -como lo es en general para la gran mayoría de los agentes químicos-, siendo la más preocupante desde el punto de vista de salud laboral (INSST, 2017). La vía dérmica es también de interés, dado que las partículas a menor tamaño tienen mayor probabilidad de atravesar la piel (HOET; BRÜSKE-HOHLFELD; SALATA, 2004). La vía oral se asocia fundamentalmente a la ingestión involuntaria de NMMs a través de la transferencia del material desde la superficie de la mano a la boca, pudiendo potencialmente cruzar la pared intestinal, ingresar al torrente sanguíneo y posteriormente llegar a otras partes del cuerpo (BARON, 2015). También pueden pasar a través de varias partes del tracto digestivo, boca, esófago, estómago, intestino delgado, intestino grueso y, por último, serían excretadas (DATTA, 2006) .

En relación con el potencial de exposición, el conocimiento actual sobre los niveles exposición humana a NMMs en todas las etapas del ciclo de vida es todavía limitada (CLARK et al., 2012). La mayor parte de los datos disponibles se corresponden con instalaciones de producción a pequeña escala -donde se llevan a cabo el desarrollo, la síntesis y el uso de NMMs nuevo o existentes-, y que han evidenciado una falta de conocimiento sobre el potencial de exposición y los riesgos laborales.

Ding et al. realizó una revisión de la exposición de los trabajadores durante los procesos de producción y manipulación de NMMs (DING et al., 2016); encontrando que el 25\% de las publicaciones investigaban las actividades de laboratorio (DÍAZ-SOLER; 
SIBRAGEC - ELAGEC 2019 - de 23 a 25 de Outubro - LONDRINA - PR

LÓPEZ- ALONSO; MARTÍNEZ-AIRES, 2017), el 63\% lo hacían sobre procesos industriales y el $12 \%$ restante estudiaban ambos.

Existe todavía un debate sobre el tipo de métrica a utilizar para respaldar los estudios de evaluación de riesgos (JIMÉNEZ; VAN TONGEREN, 2017), así como sobre los métodos para diferenciar los NMMs de otros nanomateriales presentes en el entorno (relacionados con procesos o que ocurren naturalmente).

\subsection{Toxicidad de los NMMs}

Las investigaciones sobre el potencial de exposición a NMMs durante el uso y/o producción de productos nanoestructurados o nanoproductos son todavía limitadas (BROUWER et al., 2016; CLARK et al., 2012), incluyendo operaciones y actividades vinculadas a la aplicación de NMMs en el sector de la construcción (JONES et al., 2015). Además, la existencia de datos robustos y validados es muy limitado, sobre todo en actividades que conllevan el uso de productos aditivados con NMMs o durante el tratamiento de fin de vida de residuos -especialmente en los países productores con menor producto interior bruto (BASINAS et al., 2018) .

Los niveles de exposición se encuentran estrechamente relacionados con la forma en que se usan los NMMs, esperándose un mayor potencial de exposición para los materiales manipulados en forma de polvo (GIBSON et al., 2012). Del mismo modo, las operaciones de mecanizado de productos nanoestructurados que implican la generación de polvo o la realización de tareas que implican un desgaste del producto, aumentan el potencial de liberación de NMMs (BARON, 2015) .

A pesar del hecho de existir operaciones donde puede anticiparse una posible liberación de NMMs en sector de la construcción, la información disponible sobre los niveles de exposición a NMMs en operaciones representativas es todavía escasa. Se pueden destacar operaciones como la aplicación de pinturas en aerosol, el corte, lijado, taladrado y/o mecanizado de materiales (VAQUERO et al., 2015) o estudios en tareas específicas que implican el uso de productos de construcción aditivados con NMMs (VAN BROEKHUIZEN; VAN BROEKHUIZEN, 2009; VAQUERO et al., 2015; WEST et al.,2016). En todos ellos se observan resultados dispares por lo que siguen existiendo importantes incertidumbres con respecto al riesgo de exposición en el sector de la construcción.

\subsection{Normativa}

En la Unión Europea no existe ninguna normativa específica en materia preventiva para trabajar con nanomateriales. Sin embargo, en concreto para el sector de la construcción, distintos países han publicado documentos específicos, aunque no vinculantes ni de obligado cumplimiento.

En España, el INSST ha desarrollado distintas herramientas técnicas y manuales. El más recientemente aportando nueva información sobre los niveles de exposición en procesos relevantes en el sector de la construcción (INSST, 2017).

También destacamos documentos de otros países, por ejemplo, en Reino Unido la Institution Occupational Safety and Health (IOSH) ha publicado dos documentos específicos para el sector de la construcción: Nanotechnology in construction and demolition. Guidance for industry (IOSH, 2017) y Nanotechnology in construction and demolition: what we know, what we don't (GIBB et al., 2018). En estos documentos, encontramos información detallada de las aplicaciones actuales de los NMMs en el sector 
de la construcción, sin embargo, no aportan información sobre aspectos toxicológicos o la probabilidad de exposición. Por su parte, el Instituto de Seguridad Laboral alemán (BAuA) tiene una página web interactiva donde trata esta temática (NANORAMA BAU, 2019). Finalmente, con carácter general, la Unión Europea publicó el documento Working Safely with Manufactured Nanomaterials (EUROPEAN COMMISSION, 2014), donde se incluyen recomendaciones para el uso seguro de nanomateriales en actividades enmarcadas en el sector de la construcción.

\section{NORMAS DE SEGURIDAD EN EL USO DE NMMS EN CONTRUCCIÓN}

Una de las herramientas que los técnicos de prevención de riesgos tienen son las Fichas de Seguridad de los Productos, pero actualmente dichas fichas carecen en su mayoría tanto de información sobre la presencia de NMMs en la formulación de los productos, como de información sobre medidas de gestión específica (DÍAZ-SOLER; MARTÍNEZ- AIRES; LÓPEZ-ALONSO, 2019).

Los documentos comentados en el apartado anterior concluyen en la aplicación de las normas básicas que se aplican en la Higiene Industrial (DÍAZ-SOLER; MARTÍNEZAIRES; LÓPEZ-ALONSO, 2016), insistiendo en la necesidad de información y formación tanto de técnico como de trabajadores. Todo ellos enmarcado el un principio fundamental: EL PRINCIPO DE PRECAUCIÓN (ver Figura 2).

\section{Figura 2- Introducción a la nanotecnología y NMs}

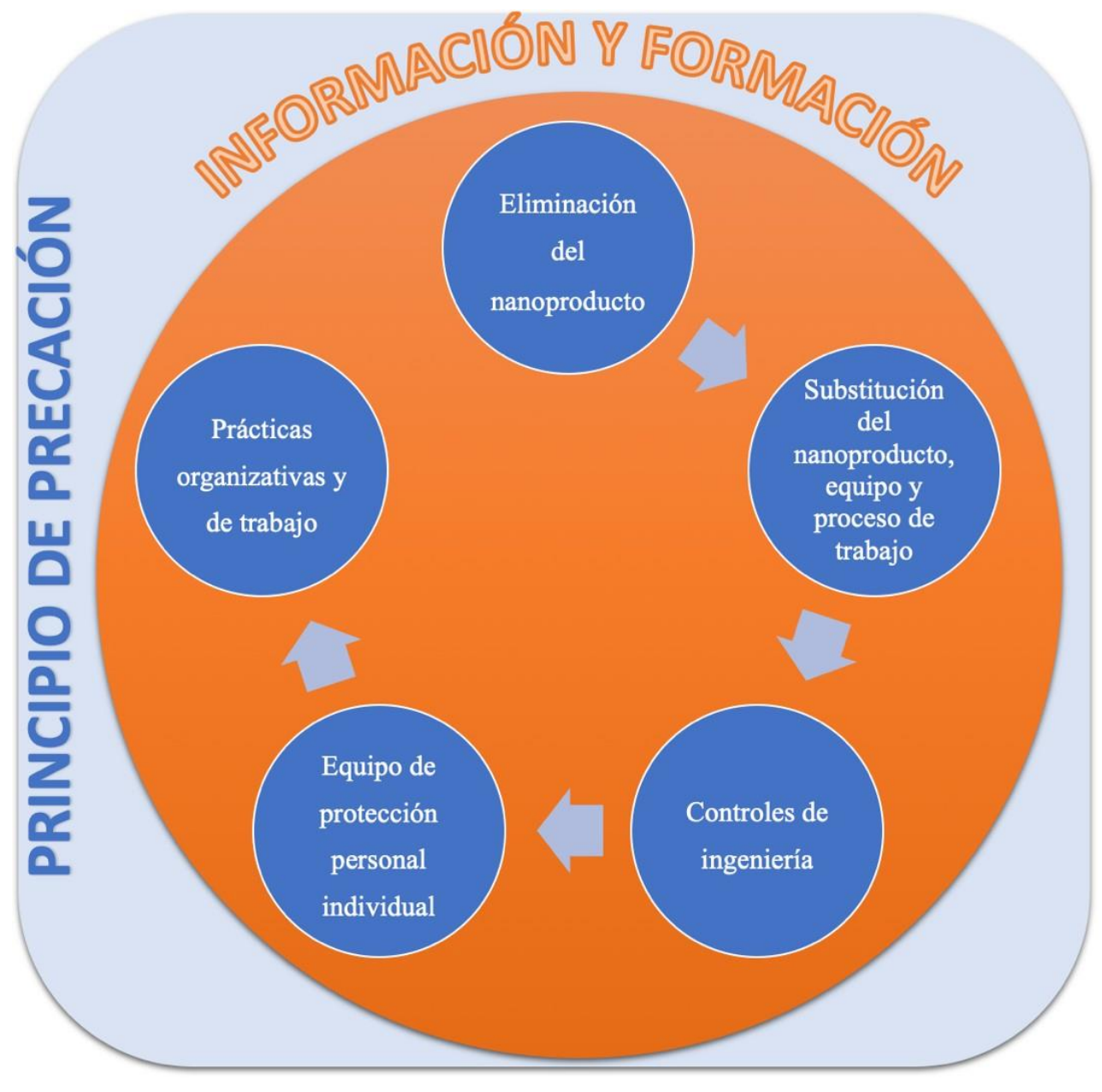


SIBRAGEC - ELAGEC 2019 - de 23 a 25 de Outubro - LONDRINA - PR

\section{CONCLUSIONES YCONSIDERACIONES FINALES}

Hoy en día, la nanotecnología es un motor de la innovación tecnológica para distintos sectores industriales. En el sector de la construcción los NMMs ofrecen un gran abanico de ventajas, proporcionando a los materiales características técnicas como mayor durabilidad, resistencia, conductividad eléctrica, etc.

Por otro lado, existe una falta de información sobre los riesgos específicos de los NMMs en los productos y actividades de relevancia en el sector de la construcción, así como sobre medidas específicas para su gestión y control por parte de las personas con responsabilidades en materia preventiva. Todo ello hace necesario investigar en las actuales prácticas preventivas utilizadas por parte del personal que intervine a lo largo el ciclo de vida de los NMMs.

Frente a este nuevo riesgo emergente, la gestión de la prevención de los riesgos derivados del uso de NMMs debe llevarse a cabo aplicando los principios generales de la Higiene Industrial, sin olvidar, el Principio de Precaución.

\section{REFERENCIAS}

AECOM. The nanotechnology energy revolution, 2014. Disponible en:https://www.aecom.com/blog/the-nanotechnology-energy-revolution/. Acceso: 13 de marzo de 2019.

BARON, M. Safe handling of nano materials and other advanced materials at workplaces. Disponible en: http://www.nanovalid.eu. Acceso: 2015.

BASINAS, I.; JIMÉNEZ, A. S.; GALEA, K. S.; VAN TONGEREN, M.; HURLEY, F. A systematic review of the routes and forms of exposure to engineered nanomaterials. Annals of Work Exposures and Health, v 62, n. 6, p. 639-662. 2018.

BROUWER, D.; SPAAN, S.; ROFF, M.; SLEEUWENHOEK, A.; TUINMAN, I.; GOEDE, H.; VAN DUUREN-STUURMAN, B.; FILON, F. L.;BELLO, D.; CHERRIE, J. W. Occupational dermal exposure to nanoparticles and nano-enabled products: Part 2, exploration of exposure processes and methods of assessment. International journal of hygiene and environmental health, v. 219, n. 6, International journal of hygiene and environmental health, p. 503-512, 2016.

CLARK, K.; VAN TONGEREN, M.; CHRISTENSEN, F. M.; BROUWER, D.; NOWACK, B.; GOTTSCHALK, F.; RIEDIKER, M. Limitations and information needs for engineered nanomaterial-specific exposure estimation and scenarios: Recommendations for improved reporting practices. Journal of Nanoparticle Research, v.14, n. 9. 2012.

DATTA, K. Current knowledge about nanotechnology safety. RAMS '06. Annual Reliability and Maintainability Symposium. 2006.

DÍAZ SOLER, B. M. Análisis integral de la seguridad y salud laboral en el uso de nanomateriales en edificación, 2019. Tesis doctoral - Programa de Doctorado de Ingeniería Civil de la Universidad de Granada.

DÍAZ-SOLER, B. M.; LÓPEZ-ALONSO, M.; \& MARTÍNEZ-AIRES, M. D. Nanosafety practices: Results from a national survey at research facilities. Journal of Nanoparticle Research, v. 19, n. 5., p. 169. 2017.

DÍAZ-SOLER, B. M.; MARTÍNEZ-AIRES, M. D.; \& LÓPEZ-ALONSO, M. Emerging risk in the construction industry: Recommendations for managing exposure to nanomaterials. [Riesgo emergente en la industria de la construcción: Recomendaciones para controlar la exposición a nanomateriales] DYNA (Colombia), v. 83, n. 196, p. 48-54. 2016. 


\section{SIBRAGEC - ELAGEC 2019 - de 23 a 25 de Outubro - LONDRINA - PR}

DÍAZ-SOLER, B. M.; MARTÍNEZ-AIRES, M. D.; \& LÓPEZ-ALONSO, M. Potential risks posed by the use of nano-enabled construction products: A perspective from coordinators for safety and health matters. Journal of Cleaner Production, n. 220, p. 33-44. 2019.

DING, Y.; STAHLMECKE, B.; KAMINSKI, H.; JIANG, Y.; KUHLBUSCH, T. A. J.; \& RIEDIKER, M. Deagglomeration testing of airborne nanoparticle agglomerates: Stability analysis under varied aerodynamic shear and relative humidity conditions. Aerosol Science and Technology, v. 50, n. 11, p. 1253-1263. 2016.

EUROPEAN COMMISSION. Working safely with manufactured nanomaterials. Guidance for workers. 2014. Disponible en: http://ec.europa.eu/social/BlobServlet?docId=13088\&langId=en. Acceso en: enero de 2019.

GIBB, A.; JONES, W.; GOODIER, C.; BUST, P.; SONG, M.; \& JIN, J. Nanotechnology in construction and demolition: What we know, what we don't. Construction Research and Innovation, v. 9, p. 55-68. 2018.

GIBSON, R.; STACEY, N.; DRAIS, E.; WALLIN, H.; ZATORSKI, W. Risk perception and risk communication with regard to nanomaterials in the workplace. 2012. Disponible en: https://osha.europa.eu/es/tools-and- publications/publications/literature_reviews/. Acceso en: enero de 2019.

HOET, P. H. M.; BRÜSKE-HOHLFELD, I.; \& SALATA, O. V. Nanoparticles - known and unknown health risks. Journal of Nanobiotechnology, v. 2. 2014.

INSST. Exposición potencial a nanomateriales en el sector de la construcción. Madrid. 2017. Disponible en: https://www.insst.es/documents/94886/538970/Exposición+potencial+a+nanomateriales+en+el +sector+de+la+construcción.pdf. Acceso en: enero de 2019.

IOSH. Nanotechnology in construction and demolition. Guidance for industry. 2017. Disponible en: https://www.iosh.com/media/1520/nanotechnology-in-constructionanddemolition-guidance.pdf. Acceso en: enero de 2019.

JIMÉNEZ, A. S.; VAN TONGEREN, M. Assessment of human exposure to ENMs. Advances in Experimental Medicine and Biology, v 947, 2017, p. 27-40. 2017.

JONES, W.; GIBB, A.; GOODIER, C.; BUST, P.; JIN, J.; \& SONG, M. Nanomaterials in construction and demolition - how can we assess the risk if we don't know where they are? Journal of Physics: Conference Series, v. 617, n. 1. 2015.

NANORAMA BAU. Disponible en: http://nano.dguv.de/nanorama/bgbau/. Acceso en: $10 \mathrm{de}$ marzo de 2019.

STATNANO. Disponible en: https://statnano.com. Acceso en: 10 de marzo de 2019.

VAN BROEKHUIZEN, P. Dealing with uncertainties in the nanotech workplace practice: Making the precautionary approach operational. Journal of Biomedical Nanotechnology, v. 7, n. 1, p. 15-17. 2011.

VAQUERO, C.; JÓPEZ, J.; STOCKMANN, H.; VAANANEN, V. Best practice guide for risk assessment of manufactured nanomaterials (MNMs) in the construction sector. Scaffold SPD9. 2015.

WEST, G. H.; LIPPY, B. E.; COOPER, M. R.; MARSICK, D.; BURRELLI, L. G.; GRIFFIN, K. N.; \& SEGRAVE, A. M. Toward responsible development and effective risk management of nano-enabled products in the U.S. construction industry. Journal of Nanoparticle Research, v. 18, n. 2, p. 1-27. 2016. 